\title{
The Place Importance of Serologic Techniques in Tuberculosis Diagnosis
}

\author{
Gülnur Tarhan* \\ Department of Medical Microbiology, Adlyaman University, Turkey \\ *Corresponding author: Gülnur Tarhan, Faculty of Medicine, Department of Medical Microbiology, Adıyaman University, Turkey
}

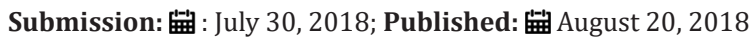

\begin{abstract}
Diagnosing active TB accurately and rapidly is a key challenge for eradicating the TB epidemic.Conventional culture methods are slow and staining methods not sufficiently sensitive. Nucleic acid amplification techniques (NAATs) tend to be costly and in some cases lack sensitivity. Antibody detection tests (serological tests) have a long history and have been used successfully for the rapid diagnosis of many infectious diseases (e.g., HIV, syphilis, and viral hepatitis). TB serological tests almost exclusively rely on antibody recognition of antigens of Mycobacterium tuberculosis by the humoral immune response, as opposed to antigen recognition by the cellular immune response (e.g. interferon-gamma release assays. These tests use various modifications of enzyme-linked immunosorbent assay (ELISA) or immunochromatographic methods to detect different antibody classes. Cellular immunodiagnostics, including tuberculin skin test (TST) and Interferon-Gamma Release Assays (IGRAs) have been used to diagnose latent tuberculosis infection (LTBI). The TST is the only universally accepted test for the diagnosis of LTBI.
\end{abstract}

Keywords:Tuberculosis; Diagnosis; Serological tests

\section{Introduction}

Tuberculosis (TB) is an important global public health problem all over the world. Diagnosing active TB accurately and rapidly is a key challenge for eradicating the TB epidemic [1-3]. Traditional culture methods are slow and staining methods not sufficiently sensitive. Nucleic acid amplification techniques (NAATs) tend to be costly and in some cases lack sensitivity [4-10]. Antibody detection tests (serological tests) have a long history and have been used successfully for the rapid diagnosis of many infectious diseases (e.g., HIV, syphilis, and viral hepatitis) [11-14]. These test are based on the patients' immune response to TB antigens. Previous or current infections may result in positive response depending on the patients' immune status.TB serological tests almost exclusively rely on antibody recognition of antigens of Mycobacterium tuberculosis by the humoral immune response, as opposed to antigen recognition by the cellular immune response (e.g. interferon-gamma release assays) $[15,16]$. Different mycobacterial proteins have been used for diagnosis of of TB. These tests use various modifications of enzyme-linked immunosorbent assay (ELISA) or immunochromatographic methods to detect different antibody classes(mostly IgG) [17-20]. Compared to convantional methods (microscopy,culture); antibody detection methods may enable rapid TB diagnosis, as these tests have the advantages of being quick and technologically easy, requiring minimal training. In addition, these tests could be adapted to point-of-care formats that can be implemented at lower levels of health services in low- and middle-income countries $[15,16]$.

Although detection of antibodies to Mycobacterium tuberculosis complex in the blood is a relatively simple and costeffective, the existing serological tests for TB are inaccurate to diagnosis pulmonary TB as well as extrapulmonary TB. Thus, the WHO issued a policy statement against the use of serological tests for the diagnosis of active TB [17]. No serological TB test is recommended by international guidelines for clinical use nor approved by the US Food and Drug Administration, dozens of distinct commercial serological tests are marketed in many parts of the world, especially in developing countries with weak regulatory systems.Cellular immunodiagnostics, including tuberculin skin test (TST) and Interferon-Gamma Release Assays (IGRAs) have been used to diagnose latent tuberculosis infection (LTBI). The TST is the only universally accepted test for the diagnosis of LTBI. However, sensitivity of TST can be compromised in individuals with immunodeficiency; and specificity can be affected by previous BCG vaccination and exposure to non-tuberculosis mycobacterium. Furthermore, subjectivity of its interpretation, the need for a return visit, and the fact that TST does not distinguish latent and active TB limits the usefulness of TST Compared to TST, IGRAs have several advantages including superior specificity and sensitivity, 
requirement of a single visit, and it does not cause bias to read the test result [18-20].

\section{Immunocromatographic tests (ICT)}

The ICT Tuberculosis test is a rapid, card-based immunochromatographic test for detection of antibodies directed against a variouspurified $\mathrm{M}$. tuberculosis antigens immobilized in lanes on a test strip and generally uses an anti-human IgG labeled with colloidal gold [21-23]. In 2005, the UNICEF/UNDP/ World Bank/WHO Special Programme for Research and Training in Tropical Diseases (TDR) performed an evaluation of 19 commercially available rapid diagnostic TB tests. The evaluation reported that, in comparison with culture plus clinical follow-up, commercial tests provided sensitivity and specificity values of $1 \%$ to $60 \%$ and $53 \%$ to $99 \%$, respectively [24].

\section{Antibody- based tests for detection TB}

There are numerous commercial antibody based serological tests for TB diagnostics.Different mycobacterial proteins have been used for serological diagnosis of TB. Initially complex antigens like culture filtrate and later on single purified antigens like Ag85 are being used for this purpose [15,21,22]. Advancement in molecular biology techniques have identified novel antigens of M. tuberculosis, some of them have been used to detect specific antibodies raised against them in TB patients. In order to develop a sensitive and a specific serodiagnostic test for TB, purified $M$. tuberculosis secreted proteins were tested to check their potential for use in the serodiagnosis.The antigens used were antigen 85a (ag85a), antigen 85b (ag85b), antigen 85c (ag85c), culture filtrate protein-10 kiloDalton (CFP-10), early secretary antigenic target-6 kiloDalton (ESAT-6) and heat shock proteins (HSP), MPT64. WHO has reviewed 67 studies for pulmonary TB and 25 studies for extrapulmonary TB in which serological tests have been evaluated. The sensitivity and specificity of serological tests have varied from $0 \%$ to $100 \%$ and from $31 \%$ to $100 \%$, respectively. These tests are not recommended for use in TB diagnostics [25-33].

\section{Interferon-gamma release assays ( IGRA Test)}

IGRAs are in vitro immune tests based on the detection of antigen-specific $\mathrm{T}$ cell immune responses. The methods have been introduced as an alternative to TST for the diagnosis of latent tuberculosis infection (LTBI) $[34,35]$. Two commercial IGRA tests are available. QuantiFERON@-TB Gold In-Tube (QFT-IT) (Qiagen, Germany) quantitates released IFN- $\gamma$ from the supernatant after whole-blood antigen stimulation using an enzyme-linked immunosorbent assay (ELISA).T-SPOT®.TB (Oxford Immunotec Ltd, Abingdon, UK) measures the frequencies of antigen-specific IFN- $\gamma$-producing cells from a purified lymphocyte fraction with an enzyme-linked immune spot assay (ELISPOT) (35). Both assays use the following M. tuberculosis-specific antigens, the early secretory antigenic target-6 (ESAT-6), the culture filtrate protein-10 (CFP10), and TB7.7 (p4) (only in QFT-IT), which all are absent in most of the NTMs and BCG strains. This feature is advantageous over PPD, especially in populations with a high NTM exposure and general BCG vaccination. Neither of the IGRAs can distinguish between active TB and LTBI [17-20]. The TB Network European Trials Group and ECDC have carried out systematic reviews and meta-analyses to assess the accuracy of IGRAs in the diagnosis of active TB and LTBI in different populationsAs concluded in the ECDC guidance, no added value of IGRAs combined with standard methods for active TB diagnostics has been found [20]. However, in certain clinical situations, including patients with EPTB or negative AFB staining or culture, children, or the differential diagnosis of infection with NTM, IGRAs can be used to supplement the diagnostic work-up. IGRAs should not be used as a rule-out test of active TB. For the diagnosis of LTBI in low-incidence countries, clear advantages over TST have been shown, and IGRAs can be used in contacttracing algorithms and in risk assessment to identify individuals for preventative treatment. A negative IGRA result does not rule out LTBI. Only subjects who have an increased risk of developing active TB from LTBI, and would benefit from preventative therapy, should be tested by IGRA. The estimated sensitivity of IGRAs for the detection of LTBI is $80-90 \%$, when culture-confirmed TB patients have been tested. In low TB settings, the specificity of over $95 \%$ has been identified. The reproducibility of the IGRAs is limited and highly susceptible to numerous factors related to manufacturer, sample processing, analytical testing and immunological variability [36-40].

\section{References}

1. Keeler E, Perkins MD, Small P, Hanson C, Reed S (2006) Reducing the global burden of tuberculosis: The contribution of improved diagnostics. Nature 444(1): 49-57

2. $\mathrm{h} t \mathrm{t} p: / / \mathrm{a} p \mathrm{p}$. $\mathrm{w}$ h o . i $\mathrm{n}$ t / i $\mathrm{r}$ i $\mathrm{s} /$ bitstream/10665/91355/1/9789241564656_eng.pdf.

3. (2013) Centers for disease control and prevention (CDC), division of tuberculosis elimination. Core Curriculum of Tuberculosis.

4. Young DB, Perkins MD, Duncan K, Barry CE (2008) Confronting the scientific obstacles to global control of tuberculosis. J Clin Invest 118(4): 1255-1265.

5. Siddiqi K, Lambert ML, Walley J (2003) Clinical diagnosis of smearnegative pulmonary tuberculosis in low-income countries: The current evidence. Lancet Infect Dis 3(5): 288-296.

6. Steingart KR, Ng V, Henry M, Hopewell PC, Ramsay A (2006) Sputum processing methods to improve the sensitivity of smear microscopy for tuberculosis: A systematic review. Lancet Infect Dis 6(10): 664-674.

7. Mase SR, Ramsay A, Ng V, Henry M, Hopewell PC (2007) Yield of serial sputum specimen examinations in the diagnosis of pulmonary tuberculosis: A systematic review. Int J Tuberc Lung Dis 11(5): 485-495.

8. Squire SB, Belaye AK, Kashoti A, Salaniponi FM, Mundy CJ (2005) 'Lost' smear-positive pulmonary tuberculosis cases: Where are they and why did we lose them? Int J Tuberc Lung Dis 9(1): 25-31.

9. World Health Organization (2013) Treatment of tuberculosis: Guidelines for national programmes. WHO ( $\left.3^{\text {rd }} \mathrm{edn}\right)$, pp. 1-113.

10. Perkins MD, Roscigno G, Zumla A (2006) Progress towards improved tuberculosis diagnostics for developing countries. Lancet 367(9541): 942-943.

11. Daniel TM (1998) Antibody and antigen detection for the immunodiagnosis of tuberculosis: Why not? what more is needed? where do we stand today? J Infect Dis 158(4): 678-680.

12. Gennaro ML (2000) Immunologic diagnosis of tuberculosis. Clin Infect Dis 30(3): 243-246. 
13. Steingart KR, Henry M, Laal S (2007) Commercial serological antibody detection tests for the diagnosis of pulmonary tuberculosis: a systematic review. PLoS Med 4(6): 202.

14. Steingart KR, Henry M, Laal S (2007) A systematic review of commercial serological antibody detection tests for the diagnosis of extrapulmonary tuberculosis. Postgrad Med J 83(985): 705-712.

15. Bothamley GH (1995) Serological diagnosis of tuberculosis. Eur Respir J 20: 676-688.

16. Verbon A, Weverling GJ, Kuijper S, Speelman P, Jansen HM (1993) Evaluation of different tests for the serodiagnosis of tuberculosis and the use of likelihood ratios in serology. Am Rev Respir Dis 148(2): 378-384.

17. World Health Organization (WHO) (2011) Policy statement. Commercial serodiagnostic tests for diagnosis of tuberculosis.

18. Centers for Disease Control and Prevention (CDC) (2013) Targeted tuberculin testing and treatment of latent tuberculosis infection.

19. Rose MV, Kimaro G, Nissen TN, Kroidl I, Hoelscher M, et al. (2012) QuantiFERON®-TB gold in-tube performance for diagnosing active tuberculosis in children and adults in a high burden setting. PLoS One 7(7): e37851.

20. European Centre for Disease Prevention and Control (ECDC) Guidance (2011) Use of interferon-gamma release assays in support of TB diagnosis.

21. Cole RA, Lu HM, Shi YZ, Wang I, De-Hua T, et al. (1996) Clinical evaluation of a rapid immunochromatographic assay based on the $38 \mathrm{kDa}$ antigen ofMycobacterium tubercu1 osis on patients with pulmonary tuberculosis in China. Tuberc Lung Dis 77(4):363-368.

22. Daniel TM (1990) The rapid diagnosis of tuberculosis: laboratory techniques applicable in developing country settings. Rev Infect Dis 11(Suppl 2): 471-478.

23. Hasegawa N, Miura T, Ishii K, Yamaguchi K, Lindner TH, et al. (2002) New simple and rapid test for culture confirmation of Mycobacterium tuberculosis complex: a multicenter study 40(3): 908-912.

24. World Health Organization (WHO) (2008) Laboratory-based evaluation of 19 commercially available rapid diagnostic tests for tuberculosis (Diagnostics evaluation series, 2).

25. Steingart KR, Dendukuri N, Henry M (2009) Performance of purified antigens for serodiagnosis of pulmonary tuberculosis: a meta-analysis. Clin Vaccine Immunol 16(2): 260-276.

26. Simonney N, Molina JM, Molimard M, Oksenhendler E, Perronne C (1995) Analysis of the immunological humoral response to mycobacterium tuberculosis glycolipid antigens (DAT, PGLTb1) for diagnosis of tuberculosis in HIV-seropositive and -seronegative patients. Eur J Clin Microbiol Infect Dis 14: 883-891.

27. Ottenhoff TH, Ellner JJ, Kaufmann SH (2012) Ten challenges for TB biomarkers. Tuberculosis(Edinb) 92 Suppl 1: 17-20
28. Leyten EM, Lin MY, Franken KL, Friggen AH, Prins C, et al. (2006) Human T-cell responses to 25 novel antigens encoded by genes of the dormancy regulon of Mycobacterium tuberculosis. Microbes Infect 8(8): 20522060.

29. Black GF, Thiel BA, Ota MO, Parida SK, Adegbola R, et al. (2009) GCGH Biomarkers for TB Consortium. Immunogenicity of novel DosR regulonencoded candidate antigens of Mycobacterium tuberculosis in three high-burden populations in Africa. Clin Vaccine Immunol 16: 1203-1212.

30. Chegou NN, Black GF, Loxton AG, Stanley K, Essone PN, et al. (2012) Potential of novel Mycobacterium tuberculosis infection phasedependent antigens in the diagnosis of TB disease in a high burden setting. BMC Infect Dis 12: 10

31. Wang S, Chen J, Zhang Y, Diao N, Zhang S, et al. (2013) Mycobacterium tuberculosis region of difference (RD) 2 antigen Rv1985c and RD11 antigen Rv3425 have the promising potential to distinguish patients with active tuberculosis from M. bovis BCG-vaccinated individuals. Clin Vaccine Immunol 20(1): 69-76.

32. Kashyap RS, Rajan AN, Ramteke SS, Agrawal VS, Kelkar SS, et al. (2007) Diagnosis of tuberculosis in an Indian population by an indirect ELISA protocol based on detection of Antigen 85 complex: a prospective cohort study. BMC Infect Dis 7: 74.

33. Grenier J, Pinto L, Nair D, Steingart K, Dowdy D, et al. (2012) Widespread use of serological tests for tuberculosis: data from 22 high-burden countries. Eur Respir J 39(2): 502-505.

34. Chegou NN, Black GF, Kidd M, van Helden PD, Walzl G (2009) Host markers in QuantiFERON supernatants differentiate active TB from latent TB infection: preliminary report. BMC Pulm Med 9: 21-29.

35.Zuber B, Levitsky V, Jonsson G, Paulie S, Samarina A, et al. (2005) Detection of human perforin by ELISpot and ELISA: ex vivo identification of virus-specific cells. J Immunol Methods 302(1-2): 13-25.

36. Pai M, Denkinger CM, Kik SV, Rangaka MX, Zwerling A, et al. (2014) Gamma interferon release assays for detection of Mycobacterium tuberculosis infection. Clin Microbiol Rev 27(1): 3-20.

37. Schuck SD, Mueller H, Kunitz F, Neher A, Hoffmann H, et al. (2009) Identification of T-cell antigens specific for latent Mycobacterium tuberculosis infection. PLoS One 4(5): e5590.

38. Barry CE , Boshoff HI, Dartois V, Dick T, Ehrt S, et al. (2009) The spectrum of latent tuberculosis: rethinking the biology and intervention strategies. Nat Rev Microbiol 7(12): 845-855.

39. Hougardy JM, Schepers K, Place S, Drowart A, Lechevin V, et al. (2007) Heparin-binding-hemagglutinin-Induced IFN-gamma release as a diagnostic tool for latent tuberculosis. PLoS ONE 2(10): e926.

40. Sester M, Sotgiu G, Lange C, Giehl C, Girardi E, et al. (2011) Interferongamma release assays for the diagnosis of active tuberculosis: a systematic review and meta-analysis. Eur Respir J 37(1): 100-111.
Creative Commons Attribution 4.0 International License

For possible submissions Click Here

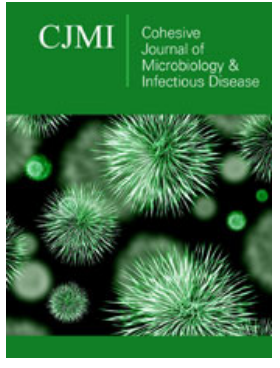

Cohesive Journal of Microbiology \& Infectious Disease

\section{Benefits of Publishing with us}

- High-level peer review and editorial services

- Freely accessible online immediately upon publication

- Authors retain the copyright to their work

- Licensing it under a Creative Commons license

- Visibility through different online platforms 